\title{
NOETHERIAN RING EXTENSIONS WITH TRACE CONDITIONS
}

\author{
ROBERT B. WARFIELD, JR.
}

\begin{abstract}
Finite ring extensions of Noetherian rings with certain restrictions on the corresponding trace ideals are studied. This setting includes finite free extensions and extensions arising from actions of finite groups when the order of the group is invertible. In this setting we establish the following results which were previously obtained (for finite extensions without trace conditions) only under strong restrictions on the rings involved. Let $R \subset S$ be an extension of Noetherian rings such that $S$ is finitely generated as a left $R$-module and such that the left trace ideal of $S$ in $R$ is equal to $R$. If $S$ is right fully bounded, or is a Jacobson ring, then $R$ has the same property; furthermore, $R$ and $S$ have the same classical Krull dimension. If $S$ is finitely generated as both a right and a left $R$-module, if both trace ideals of $S$ in $R$ are equal to $R$, and if $S$ satisfies the strong second layer condition, then this condition also holds in $R$. Finally, we compare the link graphs of $R$ and $S$.
\end{abstract}

\section{INTRODUCTION}

Many applications of Noetherian rings depend on a transfer of information between a Noetherian ring $S$ and a Noetherian subring $R$ such that $S$ is finitely generated as an $R$-module on at least one side. (For brevity we will refer to such ring embeddings as finite extensions.) Instances include embeddings of group algebras $k H \subset k G$ where $H$ is a subgroup of finite index in $G$, scalar extensions $R \subset K \otimes_{k} R$ where $R$ is a $k$-algebra and $k \subset K$ is a finite field extension, fixed rings and skew group rings $S^{G} \subset S \subset S * G$ where $G$ is a finite group of automorphisms of $S$, and embeddings of enveloping algebras $U\left(\mathbf{g}_{0}\right) \subset U(\mathbf{g})$ where $\mathbf{g}=\mathbf{g}_{0} \oplus \mathbf{g}_{1}$ is a finite-dimensional Lie superalgebra. Such applications have motivated the search for general transfer theorems for finite extensions of Noetherian rings. (See for example [5; 6, Chapters 7, 10, 12; 13; $14 ; 15$, Chapter $10 ; 17 ; 18]$.) However, many results of this nature either require further hypotheses on the internal structure of the rings involved (e.g., that the rings satisfy the second layer condition) or place additional restrictions on the embedding (e.g., that the extension ring be free as a right module over the base

Received by the editors June 14, 1990.

1991 Mathematics Subject Classification. Primary 16P40; Secondary 16D20, 16D30, 16D60, $16 \mathrm{P} 60$.

Key words and phrases. Noetherian ring, ring extension, Noetherian bimodule, trace ideal, Jacobson ring, Krull dimension, fully bounded ring, second layer condition, link, prime ideal.

This research was partially supported by a grant from the National Science Foundation. The final draft of this paper was prepared by K. R. Goodearl and E. S. Letzter from the original notes of the author. 
ring). The goal of this paper is to develop a transfer theory for finite extensions of Noetherian rings under assumptions which unite the above examples.

Our focus is primarily on the following setting: Let $R \subset S$ be an extension of Noetherian rings such that $S$ is finitely generated as a right $R$-module and such that the trace ideal of $S$ viewed as a right $R$-module is equal to $R$. (Recall that the right trace ideal of a right $R$-module $M$ is the ideal in $R$ obtained by summing the images $f(M)$ over all $f \in \operatorname{Hom}\left(M_{R}, R_{R}\right)$.) This situation encompasses in particular the case of fixed rings $R=S^{G}$ for a finite group $G$ of automorphisms of $S$ such that $|G|$ is invertible in $S$, as well as the case where $S$ is free as a right $R$-module. Moreover, many of our results are proved in more general and symmetric settings involving Noetherian bimodules with trace conditions. These bimodule results allow information to be passed from $S$ to $R$ just as well as from $R$ to $S$, in part because the $S$ - $R$-bimodule ${ }_{S} S_{R}$ automatically satisfies the condition that the left trace ideal is equal to $S$.

The information we seek to transfer between $R$ and $S$ is mostly concerned with prime ideals. In particular, we prove that the following properties descend from $S$ to $R$ : the Jacobson condition, coincidence of primitive ideals with G-ideals, and left full boundedness. When our hypotheses on the extension $R \subset S$ are left-right symmetric, the strong second layer condition descends from $S$ to $R$. (That each of these properties ascends from $R$ to $S$ is already known.) Moreover, we show that $R$ and $S$ must have the same classical Krull dimension, and we make some preliminary steps toward comparing the right Krull dimensions. Finally, we prove a lying over theorem sensitive to links between prime ideals of $R$. For this last result we assume the existence of a symmetric dimension function-such as Gelfand-Kirillov-dimension-on the prime factors of $R$ and $S$.

We now introduce our basic notation and conventions. We denote the right trace ideal in a ring $A$ of a right $A$-module $M$ by $\operatorname{Tr}\left(M_{A}\right)$ and the left trace ideal of a left $A$-module $N$ by $\operatorname{Tr}\left({ }_{A} N\right)$. When there is no confusion we will refer simply to the trace ideal of a module. Recall that a prime ideal $P$ of $S$ is said to lie over a prime $Q$ of $R$ provided $Q$ is minimal over $P \cap R$. By a Noetherian bimodule we will mean a bimodule Noetherian on each side, and by a torsionfree bimodule a bimodule torsionfree on each side. We denote the reduced rank of a module $M$ by $\rho(M)$, the classical Krull dimension of a ring $A$ by $\operatorname{clK} \operatorname{dim} A$, and the right Krull dimension of $A$ by $\mathrm{rK} \operatorname{dim} A$. Finally, for an ideal $I$ in a ring $A$, the set of elements of $A$ that are regular modulo $I$ will be denoted $\mathscr{C}_{A}(I)$ or $\mathscr{C}(I)$.

The reader is referred to $[6,9,15]$ for the definitions of unexplained terms and for background information.

\section{THE JACOBSON CONDITION}

It is proved in [5, Theorem 1] that if $V$ is a right Noetherian ring with a right Noetherian subring $U$ such that $V_{U}$ is finitely generated, then $V$ is a Jacobson ring if $U$ is a Jacobson ring. In this section we consider the converse under suitable restrictions on the right trace ideal. In [5, Theorem 2] it is shown that the converse holds when $V$ satisfies a polynomial identity, and in [14, Corollary 4.7] it is shown that the converse holds when $U$ and $V$ are Noetherian rings satisfying the second layer condition. 
Recall that if $R$ and $S$ are prime Noetherian rings and if $B$ is a nonzero $R$ - $S$-bimodule which is finitely generated and torsionfree as both a left and a right module, then $B$ is said to be a bond from $R$ to $S$ [9].

Definition 1.1. Let $R$ and $S$ be Noetherian rings and let $B$ be a Noetherian $R$ - $S$-bimodule. Let $P$ be a prime ideal of $R$, and let $Q$ be a prime ideal of $S$.

(i) If there exists a nonzero $R$ - $S$-bimodule subfactor $B^{\prime}$ of $B$ such that the left annihilator of $B^{\prime}$ is $P$, the right annihilator of $B^{\prime}$ is $Q$, and $B^{\prime}$ is a torsionfree $(R / P)-(S / Q)$-bimodule, then $B^{\prime}$ is said to be a $B$-bond from $R / P$ to $S / Q$.

(ii) Let $B\langle Q\rangle$ denote the $R$-S-bimodule preimage in $B$ of the right $(S / Q)$ torsion submodule of the right $(S / Q)$-module $B / B Q$. Alternatively, since $R$ and $S$ are Noetherian, note that

$$
B\langle Q\rangle=\bigcap\left\{\operatorname{ker}(f) \mid f \in \operatorname{Hom}\left(B_{S},(S / Q)_{S}\right)\right\} .
$$

(iii) Similarly, define $\langle P\rangle B$ to be the $R$ - $S$-bimodule preimage in $B$ of the left $(R / P)$-torsion submodule of the left $(R / P)$-module $B / P B$.

Lemma 1.2. Let $R, S$, and $B$ be as in (1.1).

(i) If $Q$ is a prime ideal of $S$, and if $\operatorname{Tr}\left(B_{S}\right) \notin Q$, then there exists a subbimodule $C<B$ such that $B / C$ is a $B$-bond from $R / P$ to $S / Q$ for some prime ideal $P$ of $R$.

(ii) If $P$ is a prime ideal of $R$, and if $\operatorname{Tr}\left({ }_{R} B\right) \nsubseteq P$, then there exists a subbimodule $C<B$ such that $B / C$ is a $B$-bond from $R / P$ to $S / Q$ for some prime ideal $Q$ of $S$.

Proof. (i) Since a right $S$-module homomorphism $f: B \rightarrow S$ can be chosen such that $f(B) \nsubseteq Q$, it follows that there exists a nonzero right $S$-module homomorphism $B \rightarrow S / Q$. Hence, $B / B\langle Q\rangle$ is not equal to zero. Let

$$
B_{0} / B\langle Q\rangle=0<B_{1} / B\langle Q\rangle<\cdots<B_{n} / B\langle Q\rangle=B / B\langle Q\rangle
$$

be a left affiliated series for the left $R$-module $B / B\langle Q\rangle$, and let $P$ be the left annihilator of $B / B_{n-1}$. Since $B / B_{n-1}$ is a torsionfree right $(S / Q)$-module [6, Proposition 7.7], it follows that $B / B_{n-1}$ is a $B$-bond from $R / P$ to $S / Q$.

(ii) This follows symmetrically.

Proposition 1.3. Let $R, B, S$, and $Q$ be as in (1.1).

(i) If $R$ is a Jacobson ring and $\operatorname{Tr}\left(B_{S}\right) \nsubseteq Q$, then $S / Q$ is semiprimitive.

(ii) If $R$ and $S / \operatorname{Tr}\left(B_{S}\right)$ are Jacobson rings, then $S$ is a Jacobson ring.

Proof. (i) It follows from 1.2(i) and the assumption of the Jacobson condition for $R$ that there exists a prime semiprimitive factor of $R$ bonded to $S / Q$. Part (i) then follows from the bond invariance of semiprimitivity [8, Theorem 6.1].

(ii) This follows directly from (i).

Corollary 1.4. Let $V$ be a Noetherian ring with a Noetherian subring $U$. If $V$ is finitely generated as a right $U$-module, and if both $U / \operatorname{Tr}\left(V_{U}\right)$ and $V$ are Jacobson rings, then $U$ is a Jacobson ring.

Proof. This follows from an application of 1.3(ii) to the bimodule ${ }_{V} V_{U}$. 


\section{Classical Characterizations OF PRIMITIVITY}

Recall that a $G$-ideal of a (Noetherian) ring $R$ is a prime ideal $P$ with the property that the intersection of all prime ideals strictly containing $P$ is strictly larger than $P$. (In other terminology, $P$ is locally closed in $\operatorname{Spec}(R)$.) Also, if $R$ is an algebra over a field $k$ and $P$ is a prime ideal of $R$ then $P$ is rational (over $k$ ) provided the center of the classical quotient ring of $R / P$ is an algebraic extension of $k$.

Let $V$ be a Noetherian ring, $U$ a Noetherian subring, and assume that $V$ is finitely generated on each side as a $U$-module. It is shown in [19, Corollary 2] that if either of the following properties holds for $U$ then the same property must also hold for $V$ : (a) each right primitive ideal is a $G$-ideal, (b) each $G$-ideal is right primitive. Moreover, if in addition $U \subseteq V$ is an extension of $k$-algebras, where $k$ is a field, then it is shown in [13, Corollary 1.5] that if one of the following properties holds for $U$ then the same property must hold for $V:$ (c) each rational prime ideal is right primitive, (d) each right primitive ideal is rational. In this section the descent of such characterizations is considered.

Proposition 2.1. Let $R, B$, and $S$ be as in (1.1).

(i) Let $P$ be a right primitive ideal of $R$ such that $\operatorname{Tr}\left({ }_{R} B\right) \notin P$. If each right primitive ideal of $S$ is coartinian then $P$ is coartinian, while if each right primitive ideal of $S$ is a G-ideal then $P$ is a G-ideal. If $R$ and $S$ are algebras over a field $k$ acting centrally on $B$, and if each right primitive ideal of $S$ is rational then $P$ is rational.

(ii) Let $Q$ be a prime idea! of $S$ such that $\operatorname{Tr}\left(B_{S}\right) \nsubseteq Q$. If each $G$-ideal of $R$ is right primitive and $Q$ is a G-ideal then $Q$ is right primitive. If $R$ and $S$ are algebras over a field $k$ acting centrally on $B$, if each rational prime ideal of $R$ is right primitive, and if $Q$ is rational, then $Q$ is right primitive.

Proof. (i) It follows from 1.2(ii) that there exists a bond from $R / P$ to $S / Q$ for some prime ideal $Q$ of $S$, and so [13, Lemma 1.3] implies that $Q$ is right primitive. It follows from [11, Proposition] that $R / P$ is artinian if $S / Q$ is artinian, from [19, Theorem 1] that $P$ is a $G$-ideal if $Q$ is a $G$-ideal, and from [3, Corollary 2.7] that $P$ is rational if $Q$ is rational.

(ii) It follows from $1.2(\mathrm{i})$ that there exists a bond from $R / P$ to $S / Q$ for some prime ideal $P$ of $R$. Part (ii) can then be deduced from a similar argument to (i), using [3, Corollary $2.7 ; 13$, Lemma 1.3].

Corollary 2.2. Let $V$ be a Noetherian ring with a Noetherian subring $U$.

(i) If $V$ is finitely generated as a right $U$-module, and each right primitive factor ring of $U$ is artinian, then each right primitive factor ring of $V$ is artinian. Moreover, if $V$ is finitely generated as a left $U$-module, if $U / \operatorname{Tr}(U V)$ is artinian, and if each right primitive factor ring of $V$ is artinian, then each right primitive factor ring of $U$ is artinian.

(ii) Suppose that $V$ is finitely generated as a left $U$-module. If either one of the following properties holds for both $V$ and $U / \operatorname{Tr}\left({ }_{U} V\right)$, then the same property also holds for $U$ : (A) each right primitive ideal is a G-ideal, (B) each right primitive ideal is rational (assuming $V$ is an algebra over a field $k$ and $U$ is a $k$-subalgebra). 
(iii) Suppose that $V$ is finitely generated as a right $U$-module. If either one of the following properties holds for both $V$ and $U / \operatorname{Tr}\left(V_{U}\right)$, then the same property also holds for $U$ : (C) each $G$-ideal is right primitive, (D) each rational prime ideal is right primitive (where $V$ is an algebra over a field $k$ and $U$ is a $k$-subalgebra).

Proof. These statements follow from applications of $(2.1)$ to the bimodules ${ }_{V} V_{U}$ and ${ }_{U} V_{V}$.

\section{BOUNDEDNESS}

Recall that a prime ring is right bounded if each essential right ideal contains a nonzero two-sided ideal. A ring is right fully bounded if each prime factor ring is right bounded.

In [18, Theorem 21] it is shown that if $U \subset V$ is a finite normalizing extension of right Noetherian rings then $U$ is right fully bounded if and only if $V$ is right fully bounded. In [14, Proposition 4.9] it is shown that if $U$ is a right fully bounded right Noetherian ring and $V$ is any ring containing $U$ as a subring such that $V_{U}$ is finitely generated, then $V$ is a right fully bounded right Noetherian ring.

Proposition 3.1. Let $R, B, S$, and $P$ be as in (1.1).

(i) If $S$ is right fully bounded and $\operatorname{Tr}\left({ }_{R} B\right) \notin P$, then $R / P$ is right bounded.

(ii) If $S$ and $R / \operatorname{Tr}\left({ }_{R} B\right)$ are right fully bounded, then $R$ is right fully bounded.

Proof. (i) It follows from 1.2(ii) that there exists a bond from $R / P$ to $S / Q$ for some prime ideal $Q$ of $S$. Assume now that $R$ and $S$ are prime and that $B$ is a bond from $R$ to $S$; to prove (i) it suffices to show that $R$ is right bounded. (We have $\operatorname{Tr}\left({ }_{R} B\right) \neq 0$ simply because $B$ is a nonzero finitely generated torsionfree left $R$-module.)

Let $I$ be an essential right ideal of $R$, and let $c$ be a regular element of $R$ contained in $I$. The regularity of $c$ ensures that $c B_{S} \cong B_{S}$. From [6, Corollary 4.18], for example, we deduce that $B / c B$ is torsion as a right $S$-module. The boundedness of $S$ implies that if $J=\operatorname{ann}(B / c B)_{S}$, then $J>0$. Consequently, $B / B J$ is not faithful as a right $S$-module, and it follows from [6, Proposition 7.4] that $B / B J$ is not faithful as a left $R$-module. Let $K=\operatorname{ann}_{R}(B / B J)$, observing that $K B \subseteq c B$.

Now let $L=\operatorname{Tr}\left({ }_{R} B\right)$. Note that $L$ is nonzero and that $L=f_{1}(B)+\cdots+$ $f_{n}(B)$ for some $f_{1}, \ldots, f_{n} \in \operatorname{Hom}\left({ }_{R} B,{ }_{R} R\right)$. Therefore,

$$
0<K L=f_{1}(K B)+\cdots+f_{n}(K B) \subseteq f_{1}(c B)+\cdots+f_{n}(c B)=c L \subseteq I .
$$

Hence $R$ is right bounded and (i) follows.

(ii) This follows from (i).

Corollary 3.2. Let $V$ be a Noetherian ring with a Noetherian subring $U$ such that $V$ is finitely generated as a left $U$-module. If $V$ and $U / \operatorname{Tr}\left({ }_{U} V\right)$ are right fully bounded then $U$ is right fully bounded.

Proof. This follows from an application of 3.1(ii) to ${ }_{U} V_{V}$. 


\section{KRULL DIMENSION}

Jategaonkar proves in [9, Theorem 8.2.8] that if $R$ and $S$ are Noetherian rings satisfying the second layer condition, and if there is a Noetherian $R-S$ bimodule which is faithful as both a left and a right module, then $R$ and $S$ have the same classical Krull dimension. In this section we show that the same conclusion can be reached if the second layer condition hypothesis is replaced with suitable trace conditions. (When $R$ is a subring of $S$ such that $S$ is finitely generated on each side as an $R$-module it can still be the case that the classical Krull dimensions of $R$ and $S$ differ-such a ring extension is constructed in $[7, \S 2]$.) We also offer some observations on how trace conditions can help to relate the right Krull dimensions of $R$ and $S$.

Lemma 4.1. Let $R$ and $S$ be Noetherian rings, and let $B$ be a Noetherian $R$-S-bimodule. Suppose that $S$ is prime and that $B$ is torsionfree as a right $S$-module. If $Q$ is a nonzero prime ideal of $S$ such that $B\langle Q\rangle \neq B$, then no left affiliated prime of $B / B\langle Q\rangle$ is minimal.

Proof. First, we may assume without loss of generality that $B$ is faithful as a left $R$-module. Let $P$ be a left affiliated prime of $B / B\langle Q\rangle$. There exists a bimodule subfactor of $B / B\langle Q\rangle$ which has annihilator equal to $P$ and which is torsionfree as a left $(R / P)$-module. Consequently, using for example [6, Lemma 10.5], we see that if $P$ is minimal then $\rho_{R}(B / B\langle Q\rangle)>0$.

Since $Q \neq 0$ there exists a regular element $c \in Q$. Note that $B c \subseteq B\langle Q\rangle$ and that $B c \cong B$ as left $R$-modules. Hence $\rho_{R}(B / B\langle Q\rangle) \leq \rho_{R}(B / B c)=0$, using for example [6, Lemma 10.4]. This inequality contradicts the previous paragraph.

Lemma 4.2. Let $R$ and $S$ be Noetherian rings, and let $B$ be a Noetherian $R-S$ bimodule such that $\operatorname{Tr}\left(B_{S}\right)=S$. If $Q$ is a prime ideal of $S$ and $B^{\prime}=B / B\langle Q\rangle$ then $\operatorname{Tr}\left(\left(B^{\prime}\right)_{S / Q}\right)=S / Q$.

Proof. Since $\operatorname{Tr}\left(B_{S}\right)=S$ we have $f_{1}(B)+\cdots+f_{n}(B)=S$ for some $f_{1}, \ldots, f_{n}$ in $\operatorname{Hom}\left(B_{S}, S_{S}\right)$. Each $f_{i}$ induces a homomorphism $g_{i} \in$ $\operatorname{Hom}\left(\left(B^{\prime}\right)_{S / Q},(S / Q)_{S / Q}\right)$, for $1 \leq i \leq n$. Further, $g_{1}\left(B^{\prime}\right)+\cdots+g_{n}\left(B^{\prime}\right)=$ $S / Q$. The lemma follows.

Theorem 4.3. Let $R$ and $S$ be Noetherian rings, and let $B$ be a Noetherian

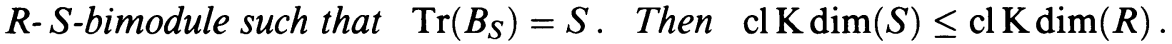

Proof. Suppose that the conclusion of the theorem is false and that $R, S$, and $B$ have been chosen such that $\alpha=\operatorname{clK} \operatorname{dim}(S)>\operatorname{clK} \operatorname{dim}(R)$ and such that $\alpha$ is minimal among classical Krull dimensions contradicting the conclusion. We may assume without loss of generality that $S$ is prime and $B_{S}$ is torsionfree, given (4.2) and the fact that the classical Krull dimension of some prime factor of $S$ will equal $\alpha$.

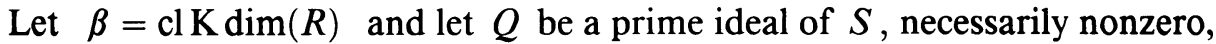
such that $\operatorname{cl} \mathrm{K} \operatorname{dim}(S / Q)=\beta$. From (4.2) it follows that $B\langle Q\rangle \neq B$. Choose a left affiliated series for ${ }_{R}(B / B\langle Q\rangle)$ with corresponding affiliated primes $P_{1}, \ldots, P_{t}$. From (4.1) it follows that none of these prime ideals is minimal. Hence if $I=\operatorname{ann}_{R}(B / B\langle Q\rangle)$ then $\operatorname{clK} \operatorname{dim}(R / I)<\beta$, and so $\operatorname{cl~K\operatorname {dim}}(S / Q)>\operatorname{cl~K\operatorname {dim}}(R / I)$. Since $\operatorname{Tr}\left([B / B\langle Q\rangle]_{S / Q}\right)=S / Q$, this contradicts the minimality of $\alpha$ and the theorem follows. 
Corollary 4.4. If $R$ and $S$ are Noetherian rings, and if there is a Noetherian $R$-S-bimodule $B$ such that $\operatorname{Tr}\left({ }_{R} B\right)=R$ and $\operatorname{Tr}\left(B_{S}\right)=S$, then $R$ and $S$ have the same classical Krull dimension.

Corollary 4.5. Let $V$ be a Noetherian ring with a Noetherian subring $U$. If $V$

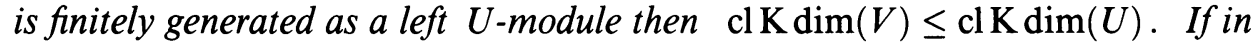
addition $\operatorname{Tr}\left({ }_{U} V\right)=U$ then $U$ and $V$ have the same classical Krull dimension.

Proof. This follows from applications of (4.3) and (4.4) to ${ }_{U} V_{V}$.

Trace conditions on a Noetherian bimodule ${ }_{R} B_{S}$ seem also to be helpful in studying the relationship between the right Krull dimensions of $R$ and $S$. We record here a few observations in this direction, stemming from discussions with K. R. Goodearl and J. T. Stafford in 1986.

Consider a Noetherian bimodule ${ }_{R} B_{S}$, and let $T=\operatorname{Tr}\left({ }_{R} B\right)$. There exist $f_{1}, \ldots, f_{n}$ in $\operatorname{Hom}\left({ }_{R} B,{ }_{R} R\right)$ such that $\sum f_{i}(B)=T$. Given any right ideal $I$ of $R$, it follows that $\sum f_{i}(I B)=I T$. Thus we can recover $I T$ from $I B$, and so if $T=R$, the map $I \mapsto I B$ provides an embedding from the lattice of right ideals of $R$ into the lattice of right $S$-submodules of $B$. Consequently,

$$
\mathrm{rK} \operatorname{dim}(S) \geq \mathrm{K} \operatorname{dim}\left(B_{S}\right) \geq \mathrm{rK} \operatorname{dim}(R)
$$

in this case. This line of argument can be adapted to the case that $\operatorname{Tr}\left({ }_{R} B\right)$ is a coartinian ideal, as follows.

Theorem 4.6. Let $R$ and $S$ be right Noetherian rings, and $B$ a nonzero $R$ $S$-bimodule such that $B_{S}$ is finitely generated. If the ideal $T=\operatorname{Tr}\left({ }_{R} B\right)$ is coartinian, then $\mathrm{rK} \operatorname{dim}(S) \geq \mathrm{rK} \operatorname{dim}(R)$.

Proof. Since $B$ is nonzero, so is $S$. Hence, the result is clear in case $R$ is right artinian. Now assume that $\mathrm{rK} \operatorname{dim}(R)>0$. It suffices to prove the following

Claim. If $I$ and $J$ are any right ideals of $R$ with $I>J$ and $\mathrm{K} \operatorname{dim}(I / J)=$ $\alpha \geq 1$, then $\mathrm{K} \operatorname{dim}(I B / J B) \geq \alpha$.

This claim reduces, by an obvious transfinite induction, to the case $\alpha=1$, and for that case we may assume that $I / J$ is 1 -critical.

Let $P$ be the assassinator (i.e., the unique associated prime) of $I / J$, and let $I^{\prime} / J=\operatorname{ann}_{I / J}(P)$. Since it is enough to show that $\mathrm{Kdim}\left(I^{\prime} B / J B\right) \geq 1$, we may replace $I$ by $I^{\prime}$. Thus we may assume that $(I / J) P=0$. Note that $\mathrm{rK} \operatorname{dim}(R / P) \geq 1$ and so $T \nsubseteq P$.

If $I_{1}$ is a right ideal of $R$ with $I \geq I_{1}>J$ and $I_{1} B=J B$, then as in the discussion above, $I_{1} T=J T$. But then $\left(I_{1} / J\right) T=0$, contradicting the fact that $T \notin P$. Thus $I_{1} B>J B$ whenever $I \geq I_{1}>J$.

If $I B / J B$ is artinian, choose a right ideal $I_{1}$ of $R$ such that $I \geq I_{1}>J$ and $I_{1} B$ is as small as possible. Then whenever $I_{2}$ is a right ideal of $R$ with $I_{1} \geq I_{2}>J$, we obtain $I_{1} B=I_{2} B$, and consequently $I_{1} T=I_{2} T \leq I_{2}$. Since $I / J$ is 1-critical, $J$ equals the intersection of the right ideals $I_{2}$ of $R$ satisfying $I_{1} \geq I_{2}>J$. But then $I_{1} T \leq J$, again a contradiction. Therefore $\mathrm{K} \operatorname{dim}(I B / J B) \geq 1$.

Corollary 4.7. Let $R$ and $S$ be Noetherian rings, and $B$ a Noetherian $R-S$ bimodule such that ${ }_{R} B$ is faithful. If all nonminimal primes of $R$ are coartinian, then $\mathrm{r} \mathrm{K} \operatorname{dim}(S) \geq \mathrm{r} \mathrm{K} \operatorname{dim}(R)$. 
Proof. Choose a minimal prime $P$ in $R$ such that

$$
\mathrm{r} \mathrm{K} \operatorname{dim}(R / P)=\mathrm{r} \mathrm{K} \operatorname{dim}(R) .
$$

The prime $P$ appears among the affiliated primes corresponding to any left affiliated series for $B$ by [6, Proposition 2.14]. Hence, $B$ has a bimodule subfactor $C$ such that $\operatorname{ann}\left({ }_{R} C\right)=P$ and $C$ is a torsionfree left $(R / P)$ module [6, Proposition 7.5]. Replace $R$ and $B$ by $R / P$ and $C$.

Thus we may assume that $R$ is prime and that ${ }_{R} B$ is torsionfree. Now the ideal $T=\operatorname{Tr}\left({ }_{R} B\right)$ is nonzero, and so all primes containing $T$ are coartinian. Therefore $T$ is coartinian, and the result follows from (4.6).

Corollary 4.8. Let $V$ be a Noetherian ring with a Noetherian subring $U$ such that all nonminimal primes of $U$ are coartinian. If $V$ is finitely generated as a left $U$-module, then $\mathrm{rK} \operatorname{dim}(V) \geq \mathrm{rKdim}(U)$. If in addition $V$ is finitely generated as a right $U$-module, then $U$ and $V$ have the same right Krull dimension.

Proof. The first conclusion follows from an application of (4.7) to the bimodule ${ }_{U} V_{V}$. In case $V_{U}$ is finitely generated, the inequality $\mathrm{r} \mathrm{K} \operatorname{dim}(V) \leq$ $\mathrm{r} \mathrm{K} \operatorname{dim}(U)$ is well known (see e.g. [15, Lemma 6.5.3(ii)(c)]).

\section{THE STRONG SECOND LAYER CONDITION}

We refer the reader to [9] or [6] for the definitions of the right and left (strong) second layer conditions, and for equivalent formulations of these conditions.

It is shown in [14, Theorem 4.2] that if $V$ is a ring containing a Noetherian subring $U$ satisfying the (strong) second layer condition such that $V$ is finitely generated as a right and left $U$-module then $V$ satisfies the (strong) second layer condition. An example in $[7, \S 2]$ shows that a simple ring $V$ (which trivially satisfies the (strong) second layer condition) may contain a fixed ring $U$ under the action of a finite group such that $U$ does not satisfy the second layer condition. Therefore a general converse does not hold. In this section we prove a converse for the strong second layer condition in the presence of trace conditions. We start with a lemma which is presumably well known, but for which we could find no reference.

Lemma 5.1. Let $R, S$, and $T$ be prime Noetherian rings. If there exist bonds from $R$ to $S$ and from $S$ to $T$, then there exists a bond from $R$ to $T$.

Proof. Let $A$ be a bond from $R$ to $S$, and $B$ a bond from $S$ to $T$. There is an isomorphism of $R$ - $T$-bimodules

$$
\operatorname{Fract}(R) \otimes_{R} A \otimes_{S} B \cong A \otimes_{S} \operatorname{Fract}(S) \otimes_{S} B \cong A \otimes_{S} B \otimes_{T} \operatorname{Fract}(T),
$$

which follows from [9, Theorem 5.2.2]. Since $A \otimes_{S} \operatorname{Fract}(S)$ and $\operatorname{Fract}(S) \otimes_{S} B$ are nonzero modules over the simple Artinian ring $\operatorname{Fract}(S)$, their tensor product is nonzero, and so the tensor products above are nonzero. If $C$ denotes the natural image of $A \otimes_{S} B$ in $\operatorname{Fract}(R) \otimes_{R} A \otimes_{S} B$, then $C$ must be nonzero, and thus $C$ is a bond from $R$ to $T$.

Lemma 5.2. Let $R, S$, and $T$ be Noetherian rings, such that $R$ and $T$ satisfy the second layer condition. Suppose that ${ }_{R} A_{S}$ and ${ }_{S} B_{T}$ are Noetherian bimodules which are faithful on each side. Assume that $S$ is prime and that 
$A_{S}$ and ${ }_{S} B$ are torsionfree. Then $R$ and $T$ possess Artinian classical quotient rings, and ${ }_{R} A$ and $B_{T}$ are torsionfree (that is, ${ }_{R} A$ is $\mathscr{C}_{R}(0)$-torsionfree and $B_{T}$ is $\mathscr{C}_{T}(0)$-torsionfree).

Proof. We first show that $R$ and $T$ possess Artinian quotient rings. In light of [9, Theorem 7.4.11] it suffices to show that the sets of minimal primes of $R$ and $T$ are link closed, that each annihilator prime of $T_{T}$ is a minimal prime, and that each annihilator prime of ${ }_{R} R$ is a minimal prime.

From (5.1) and [6, Proposition 7.7] it follows that there exist bonds from $R / P$ to $T / Q$ for each pair of minimal primes $P$ of $R$ and $Q$ of $T$. It then follows from [9, Theorem 8.2.8] that $R / P$ and $T / Q$ have the same classical Krull dimension, for any such $P$ and $Q$. Consequently, again using [9, Theorem 8.2.8], the sets of minimal primes of $R$ and of $T$ are each closed with respect to links.

We next consider the annihilator primes of ${ }_{R} A$ and $B_{T}$. Let $Q$ be a maximal annihilator prime of $B_{T}$. Then it follows from [6, Proposition 7.7] and (5.1) that there is a bond from $R / P$ to $T / Q$ for some minimal prime ideal $P$ of $R$. Hence, $R / P$ and $T / Q$ have the same classical Krull dimension, by [9, Theorem 8.2.8]. We saw above that the minimal prime factors of $R$ and $T$ all have the same classical Krull dimension, and it therefore follows that $Q$ is a minimal prime of $T$. Hence all of the annihilator primes of $B_{T}$ are minimal, and a similar argument shows that all of the annihilator primes of ${ }_{R} A$ are minimal.

Since $T_{T}$ embeds in some finite direct sum of copies of $B_{T}$, each annihilator prime of $T_{T}$ is also an annihilator prime of $B_{T}$. Thus, by the paragraph above, all of the annihilator primes of $T_{T}$ are minimal primes. A similar argument shows that each annihilator prime of ${ }_{R} R$ is minimal, and it now follows that $R$ and $T$ have Artinian quotient rings.

To see that $B_{T}$ is torsionfree, let $C$ denote the torsion submodule of $B_{T}$, and suppose that $C \neq 0$. If $D$ is a right affiliated submodule of $C$ and $P=\operatorname{ann}\left(D_{T}\right)$, then $P$ is an annihilator prime of $B_{T}$ and hence $P$ is minimal. From [6, Proposition 7.5] it follows that $D_{T / P}$ is torsionfree, and since $\mathscr{C}_{T}(0)=\mathscr{C}_{T}(N) \subseteq \mathscr{C}_{T}(P)$ (where $N$ is the prime radical of $T$ ), it follows that $D_{T}$ is torsionfree. This is impossible, and so $B_{T}$ must be torsionfree. Similar reasoning shows that ${ }_{R} A$ is torsionfree.

Lemma 5.3. Let $S$ and $T$ be Noetherian rings, and suppose that $B$ is a Noetherian $S$-T-bimodule which is faithful on each side. Suppose also that $S$ has an Artinian classical quotient ring, and that $B$ is torsionfree as a left $S$-module. Let $I$ be an ideal of $S$ not contained in any minimal prime. Then there exists an ideal $J$ of $T$, not contained in any minimal prime, such that $B J \subseteq I B$.

Proof. Since $I$ is not contained in any minimal prime of $S$, each of the primes minimal over $I$ contains an element of $\mathscr{C}_{S}(N)$, where $N$ is the prime radical of $S$. Hence, there exists an element $x \in I \cap \mathscr{C}_{S}(N)$, and $x \in \mathscr{C}_{S}(0)$ because $S$ has an Artinian quotient ring. Now $x B_{T} \cong B_{T}$, and it follows, for example from [6, Lemma 10.4], that $\rho\left((B / I B)_{T}\right)=0$. Using for example [6, Lemma 10.5], it follows that $(B / I B)_{T}$ is a $\mathscr{C}_{T}\left(N^{\prime}\right)$-torsion module, where $N^{\prime}$ is the prime radical of $T$. Now consider a right affiliated series for $B / I B$. Since $(B / I B)_{T}$ is $\mathscr{C}_{T}\left(N^{\prime}\right)$-torsion, none of its affiliated primes is a minimal prime. Hence, the ideal $J=\operatorname{ann}(B / I B)_{T}$ cannot be contained in any minimal prime of $T$, and the lemma follows. 
Lemma 5.4. Let $S$ be a prime Noetherian ring, let $T$ be a Noetherian ring with an Artinian classical quotient ring, and suppose that $T$ satisfies the right strong second layer condition. Assume that there exists a Noetherian $S$-T-bimodule $B$ which is torsionfree and faithful on each side, and such that $\operatorname{Tr}\left({ }_{s} B\right)=S$. Let $M$ be a uniform faithful cyclic right $S$-module. Then the assassinator of $M_{S}$ is zero.

Proof. Suppose that $P$ is the assassinator of $M$ and that $P \neq 0$; we will arrive at a contradiction. Let $J=\operatorname{ann}(B / P B)_{T}$. From (5.3) it follows that $J$ is not contained in any minimal prime of $T$. Without loss of generality we may assume that $M=S / K$ for some right ideal $K$ of $S$. Since $\operatorname{Tr}\left({ }_{S} B\right)=S$ it must be the case that $B / K B \neq 0$. Now choose a right ideal $L$ of $S$ containing $K$ such that $L / K=\operatorname{ann}_{M} P$. The assumption $\operatorname{Tr}\left({ }_{S} B\right)=S$ now implies that $L B / K B \neq 0$. Note that $(L B / K B) J=0$.

Choose a right $T$-submodule $C$ of $B$ containing $K B$ which is maximal such that $C \cap L B=K B$. Then $(B / C)_{T}$ is an essential extension of $((L B+C) / C)_{T}$, which is isomorphic to $(L B / K B)_{T}$. Since $T$ has an Artinian quotient ring, the set of minimal primes of $T$ is closed with respect to links. Since $J$ annihilates $(L B / K B)_{T}$, and since $T$ satisfies the right strong second layer condition, it now follows from [9, Theorem 9.1.2] that there exists an ideal $I$ of $T$, not contained in any minimal prime, such that $(B / C) I=0$ (see also [6, Theorem 11.4]). Hence, $B I \cap L B \subseteq K B$. Now let $H=\operatorname{ann}_{S}(B / B I)$. From (5.3) it follows that $H \neq 0$. However, $H B \cap L B \subseteq K B$. The assumption $\operatorname{Tr}\left({ }_{S} B\right)=S$ now implies that $H \cap L \subseteq K$. Therefore, it follows from the uniformity of $(R / K)_{S}$ that $(H+K) / K=0$, and so $M H=$ $(S / K) H=0$. The lemma follows from this contradiction to the faithfulness of $M_{S}$.

Theorem 5.5. Let $R, S$, and $T$ be Noetherian rings such that $R$ and $T$ satisfy the second layer condition, and assume that there exist Noetherian bimodules ${ }_{R} A_{S}$ and ${ }_{S} B_{T}$ such that $\operatorname{Tr}\left(A_{S}\right)=\operatorname{Tr}\left({ }_{S} B\right)=S$. If $T$ satisfies the right strong second layer condition, then so does $S$, while if $R$ satisfies the left strong second layer condition, then so does $S$.

Proof. Assume that $T$ satisfies the right strong second layer condition. If $S$ does not, then by [6, Proposition 11.3] there exists a cyclic uniform right $S$ module $M$ such that the ideal $Q=\operatorname{ann}_{S}(M)$ is prime while the associated prime $P$ of $M$ strictly contains $Q$. By (4.2),

$$
\operatorname{Tr}\left([A / A\langle Q\rangle]_{S / Q}\right)=\operatorname{Tr}(s / Q[B /\langle Q\rangle B])=S / Q \text {. }
$$

Replace $S$ by $S / Q, R$ and $T$ by $R / \operatorname{ann}_{R}(A / A\langle Q\rangle)$ and $T / \operatorname{ann}(B /\langle Q\rangle B)_{T}$, and $A$ and $B$ by $A / A\langle Q\rangle$ and $B /\langle Q\rangle B$. Thus we may assume that $S$ is a prime ring, that $A_{S}$ and ${ }_{S} B$ are torsionfree, and that ${ }_{R} A, \quad B_{T}$, and $M_{S}$ are faithful.

Now by (5.2), $T$ has an Artinian quotient ring and $B_{T}$ is torsionfree. But then from (5.4) we obtain the contradiction $P=0$. Therefore $S$ must satisfy the right strong second layer condition.

The final statement of the theorem is proved symmetrically.

Corollary 5.6. Let $V$ be a Noetherian ring satisfying the strong second layer condition and containing a Noetherian subring $U$ such that $V$ is finitely generated on each side as a $U$-module. If $\operatorname{Tr}\left(V_{U}\right)=\operatorname{Tr}\left({ }_{U} V\right)=U$ then $U$ satisfies the strong second layer condition. 
We leave as an open question whether the analog of (5.6) for the plain second layer condition holds. One case in which it does hold is when $V$ is a finite free centralizing extension of $U$; see [1, Proposition 3.18; 2, Theorem 7.8(b)].

\section{LYING OVER FOR LINKS}

In [17, Theorem 6] it is shown that if $S$ is a Noetherian algebra of finite GKdimension (Gelfand-Kirillov-dimension) containing a Noetherian subalgebra $R$ such that $S$ is finitely generated as a right $R$-module and $\operatorname{Tr}\left(S_{R}\right)=R$, then for every prime ideal $Q$ of $R$ there exists a prime $P$ of $S$ lying over $Q$. Now recall for a Noetherian ring $R$ that there exists a link from a prime $Q$ of $R$ to a prime $Q^{\prime}$ of $R$, denoted by $Q \rightsquigarrow Q^{\prime}$, provided there exists an $R$ - $R$-factor of $\left(Q \cap Q^{\prime}\right) / Q Q^{\prime} \quad$ which is torsionfree on each side as an $(R / Q)-\left(R / Q^{\prime}\right)$ bimodule. The directed graph structure thus obtained for the prime spectrum of $R$ is called the graph of links. The importance of this structure for the representation theory of $R$ is studied in [4, 12]. In this section we prove a lying over theorem for links when certain trace, finiteness, and symmetry conditions are present. (The symmetry condition will be automatically satisfied when the rings involved are algebras of finite GK-dimension.) Our results parallel [14, Theorem 5.3], where there is no trace related assumption but the presence of the second layer condition is assumed.

Lemma 6.1. Let $R$ and $S$ be Noetherian rings such that $R$ is a subring of $S$ and $S_{R}$ is finitely generated. If $Q$ is a minimal prime of $R$ there exists a prime of $S$ which lies over $Q$. Moreover, if $P$ is any prime of $S$ which lies over $Q$, there exists an $\left({ }_{S} S_{R}\right)$-bond from $S / P$ to $R / Q$.

Proof. Let $Q$ be a minimal prime of $R$. By Zorn's Lemma there is an ideal $P$ of $S$ maximal with respect to the property that $P \cap R \subseteq Q$. It is straightforward to check that $P$ is prime, and the minimality of $Q$ guarantees that $P$ lies over $Q$. (The preceding argument is well known; see for example [15, Theorem 10.2.9].) The remainder of the lemma follows from [13, Lemma 1.1] or the proof of [6, Lemma 7.15].

Lemma 6.2. Let $R$ and $S$ be Noetherian rings such that $R$ is a subring of $S$, $S_{R}$ is finitely generated, and $\operatorname{Tr}\left(S_{R}\right)=R$. Let $I$ be an ideal of $R$. Then there is an ideal $K$ of $S$ such that $K \cap R \subseteq I$ and such that if $P$ is a prime of $S$ minimal over $K$ then there is a prime $Q$ of $R$ containing $I$ with an $\left({ }_{S} S_{R}\right)$-bond from $S / P$ to $R / Q$.

Proof. Let $S\langle I\rangle=\left\{s \in S: f(s) \in I\right.$ for all $\left.f \in \operatorname{Hom}\left(S_{R}, R_{R}\right)\right\}$. (Note that this definition is consistent with 1.1(ii).) It is clear that $S\langle I\rangle$ is an $S$-R-subbimodule of $S$ and that $S I \subseteq S\langle I\rangle$. We may therefore regard $S / S\langle I\rangle$ as an $S$ - $(R / I)$-bimodule. Since $\operatorname{Tr}\left(S_{R}\right)=R$, there are elements $f_{1}, \ldots, f_{n}$ of $\operatorname{Hom}\left(S_{R}, R_{R}\right)$ such that $R=f_{1}(S)+\cdots+f_{n}(S)$. We infer that if $J$ is an ideal of $R$, then $J=f_{1}(S J)+\cdots+f_{n}(S J)$. Consequently, if $S J \subseteq S\langle I\rangle$ then $J \subseteq I$. Hence $S / S\langle I\rangle$ is faithful as a right $(R / I)$-module.

We now let $K=\operatorname{ann}_{S}(S / S\langle I\rangle)$. Note that $K$ is the largest two-sided ideal of $S$ contained within the left ideal $S\langle I\rangle$. Hence $S(K \cap R) \subseteq S\langle I\rangle$, and therefore $K \cap R \subseteq I$. Now observe that $S / S\langle I\rangle$ is an $(S / K)-(R / I)$-bimodule which is faithful and finitely generated on each side. That for each prime $P$ of $S$ minimal over $K$ there exists a prime $Q$ of $R$ such that $S / P$ is $\left({ }_{S} S_{R}\right)$ bonded to $R / Q$ now follows from [6, Propositions 2.14, 7.5, and 7.7]. 
Let $R$ be a Noetherian ring containing prime ideals $Q$ and $Q^{\prime}$. Recall that there exists an ideal link from $Q$ to $Q^{\prime}$ provided there exists an $R$-bond, where $R$ is considered as an $R$ - $R$-bimodule, from $R / Q$ to $R / Q^{\prime}$. Note that $R / Q$ provides an ideal link from $Q$ to itself. We say that there is a nontrivial ideal link from $Q$ to itself if there exists an $R$-bond from $Q$ to itself which is a subfactor of ${ }_{R} Q_{R}$. For convenience we will also denote as nontrivial any ideal link between distinct primes.

The next lemma is a refinement of [14, Theorem 5.1i].

Lemma 6.3. Let $R$ and $S$ be Noetherian rings such that $R$ is a subring of $S$. Let $Q$ and $Q^{\prime}$ be minimal prime ideals of $R$ such that there is an ideal link from $Q$ to $Q^{\prime}$.

(i) There exist primes $P$ and $P^{\prime}$ of $S$ such that $P$ lies over $Q$ and $P^{\prime}$ lies over $Q^{\prime}$, and such that there is an ideal link from $P$ to $P^{\prime}$ in $S$.

(ii) If each prime ideal of $S$ intersects to a semiprime ideal of $R$, and if the ideal link from $Q$ to $Q^{\prime}$ is nontrivial, then the primes $P$ and $P^{\prime}$ of (i) may be chosen such that the ideal link between them is nontrivial.

Proof. Suppose that the ideal link from $Q$ to $Q^{\prime}$ is given by the $R$ - $R$-bimodule $I / J$, where $I$ and $J$ are ideals of $R$. Regard $S$ as an $R$ - $R$-bimodule, and consider the series

$$
S \supset R \supset I \supset J \supset 0
$$

as a series of $R$-R-subbimodules of $S$. We also can choose a series of ideals of $S$, say

$$
S=S_{0} \supset S_{1} \supset \cdots \supset S_{n}=0,
$$

such that each factor $S_{i} / S_{i+1}$, for $0 \leq i \leq n-1$, is a bond between two primes of $S$. By the Schreier Refinement Theorem, these two series of $R$ $R$-subbimodules have isomorphic refinements. This means that there exists a factor $S_{i} / S_{i+1}$, for some $0 \leq i \leq n-1$, which has an $R$ - $R$-bimodule subfactor isomorphic to $I^{\prime} / J$, where $I^{\prime}$ is an ideal of $R$ and $I \supseteq I^{\prime} \supset J$. Let $P$ be the left $S$-annihilator of $S_{i} / S_{i+1}$, and let $P^{\prime}$ be the right $S$-annihilator of $S_{i} / S_{i+1}$. Clearly $Q \supseteq P \cap R$ and $Q^{\prime} \supseteq P^{\prime} \cap R$. Since $Q$ and $Q^{\prime}$ are minimal we conclude that $P$ lies over $Q$ and $P^{\prime}$ lies over $Q^{\prime}$. This proves part (i).

Now suppose in addition that the ideal link $I / J$ from $Q$ to $Q^{\prime}$ is nontrivial and that prime ideals of $S$ intersect to semiprime ideals of $R$. Let $N$ be the prime radical of $S$. Our hypothesis implies that the ideal $M=N \cap R$ is semiprime. Clearly $M$ is nilpotent, and so $M$ is the prime radical of $R$. Since there cannot exist a nontrivial ideal link between $Q / M$ and $Q^{\prime} / M$ in $R / M$, it follows that $(M \cap I) /(M \cap J)$ is a nontrivial ideal link from $Q$ to $Q^{\prime}$. Replace $I$ with $M \cap I$ and $J$ with $M \cap J$. Consider the series $N \supset M \supset I \supset J \supset 0$, and choose a series of ideals of $S$, say $N=N_{0} \supset$ .. $\supset N_{m}=0$, such that each factor $N_{j} / N_{j+1}$, for $0 \leq j \leq m-1$, is a bond between two primes of $S$. An argument similar to the one in the previous paragraph now proves the conclusion of (ii).

We next restrict our attention to collections of Noetherian rings which possess a certain symmetry condition.

Definition 6.4. A collection $X$ of Noetherian rings is said to possess a symmetric dimension function if there exists a function $d$ assigning to each prime 
factor ring of each ring $R \in X$ an element of a fixed totally ordered set such that $d$ satisfies the following conditions:

(i) If $P$ and $Q$ are prime ideals of a ring $R \in X$ such that $Q>P$ then $d(R / Q)<d(R / P)$.

(ii) If $R$ and $S$ are prime factors of rings in $X$, and if there exists a bond from $R$ to $S$, then $d(R)=d(S)$.

We extend such a function $d$ to arbitrary factor rings $R$ of rings in $X$ by setting $d(R)$ equal to the maximum of $d(R / P)$ for $P$ ranging over the minimal primes of $R$.

Remarks. (i) Let $X$ be a collection of algebras of finite GK-dimension over a fixed field $k$. For each prime factor $R$ of an algebra in $X$ let $d(R)$ denote the GK-dimension of $R$. It follows, for example, from [10, Corollary 3.16, Lemma 5.3, Corollary 5.4] that the dimension function $d$ satisfies properties 6.4(i) and 6.4(ii). Hence $X$ possesses a symmetric dimension function. Now extend $d$ to arbitrary factors of algebras in $X$ as in (6.4). It is an open question whether this extension of $d$ will be equivalent to GK-dimension. In other words, it is not known whether the GK-dimension of an algebra $R$ is equal to the maximum value of the GK-dimensions of $R / P$ for $P$ ranging over the minimal primes of $R$. (See [10, Chapter 5] or [15, Chapter 8] for more information.)

(ii) Note that (6.4) will be satisfied by classes $X$ of Noetherian rings for which the right Krull dimension equals the left Krull dimension in every bond between prime factors of rings in $X$. It is therefore an open question whether every collection of Noetherian rings possesses a symmetric dimension function.

Lemma 6.5. Let $S$ be a Noetherian ring possessing a symmetric dimension function $d$. Let $P$ and $P^{\prime}$ be minimal primes of $S$ such that there is an ideal link from $P$ to $P^{\prime}$. Suppose further that $d(S / P)=d\left(S / P^{\prime}\right)=d(S)$. If the ideal link from $P$ to $P^{\prime}$ is nontrivial then there exist primes $P_{1}, \ldots, P_{m}$ of $S$, with $m \geq 2$, such that $P_{1}=P$ and $P_{m}=P^{\prime}$, and such that $P_{i} \rightsquigarrow P_{i+1}$ for $1 \leq i \leq m-1$.

Proof. Suppose a nontrivial ideal link from $P$ to $P^{\prime}$ is given by $I / J$. Note that since $J$ annihilates $I / J$ on each side, $J \subseteq P \cap P^{\prime}$. We may assume without loss of generality that $J=0$. Let $K$ be an ideal of $S$ maximal among ideals whose intersection with $I$ is zero. Since $K I=I K=0$, we see that $K$ is contained within both $P$ and $P^{\prime}$ and that there is an ideal link in $S / K$ via $(I+K) / K$ from $P / K$ to $P^{\prime} / K$. So without loss of generality we may assume that $K=0$. Now let $L$ be any ideal of $S$ such that $Y L=0$ for some nonzero subset $Y$ of $S$. Because $S Y S \cap I \neq 0$, we see that $L \subseteq P^{\prime}$ since $I$ is torsionfree as a right $\left(S / P^{\prime}\right)$-module. Hence $P^{\prime}$ is the unique associated prime of $S_{S}$. Also, since the codimension of $P^{\prime}$ is equal to the dimension of $S$, and since $d$ satisfies the properties of (6.4), we see that the right clique (see [9]) of $P^{\prime}$ consists of minimal primes. Therefore, the right clique of $P^{\prime}$ satisfies the strong second layer condition.

Let $U=1 . \operatorname{ann}_{S}\left(P^{\prime}\right)$. Since $P^{\prime}$ is the only right associated prime of $S$, we see that $U$ is an essential right ideal of $S$. Since the right clique of $P^{\prime}$ satisfies the strong second layer condition and incomparability, it follows from [4, §5] or $\left[9\right.$, Theorem 9.1.2] that $(S / U) P_{n} \cdots P_{1}=0$ for some primes $P_{1}, \ldots, P_{n}$ 
such that for each $1 \leq i \leq n$ there is a nonzero sequence of links

$$
P_{i} \rightsquigarrow Q_{i 1} \rightsquigarrow Q_{i 2} \rightsquigarrow \cdots \rightsquigarrow Q_{i, m_{i}} \rightsquigarrow P^{\prime} \text {. }
$$

Note that $P_{n} \cdots P_{1} P^{\prime}=0$.

Suppose that $P \neq P^{\prime}$. Since $P_{n} \cdots P_{1} P^{\prime} \subseteq P$, it then follows for some $i$ that $P_{i} \subseteq P$. But the minimality of $P$ shows that $P=P_{i}$ and the conclusion of the lemma follows in this case. So suppose that $P=P^{\prime}$. Since the ideal link is nontrivial, $P \neq 0$ and so $P \cap I \neq 0$. Hence, $1 . \operatorname{ann}_{S}(P \cap I)=P$. Since $\left(P_{n} \cdots P_{1}\right)(P \cap I)=0$, we see that $P_{n} \cdots P_{1} \subseteq P$. The lemma follows.

Theorem 6.6. Let $S$ be a Noetherian ring containing a Noetherian subring $R$ such that $S$ is finitely generated as a right $R$-module. Suppose that $\{R, S\}$ possesses a symmetric dimension function $d$, and suppose that $\operatorname{Tr}\left(S_{R}\right)=R$. Let $Q$ and $Q^{\prime}$ be primes of $R$ such that $Q \rightsquigarrow Q^{\prime}$. Then there are primes $P_{1}, \ldots, P_{m}$ of $S$ such that $P_{1}$ lies over $Q$ and $P_{m}$ lies over $Q^{\prime}$, and such that $P_{i} \rightsquigarrow P_{i+1}$ for $1 \leq i<m$. While it may be the case that $m=1$ and no links occur, we can require that $m>1$ if each prime ideal of $S$ intersects to a semiprime ideal of $R$.

Proof. Suppose the link from $Q$ to $Q^{\prime}$ is given by the ideal factor $\left(Q \cap Q^{\prime}\right) / I$. Let $d(R / Q)=\alpha$, and note by (6.4) that $d\left(R / Q^{\prime}\right)=\alpha$. We apply (6.2), obtaining an ideal $K$ of $S$ such that $K \cap R \subseteq I \subset Q \cap Q^{\prime}$ and such that if $P$ is a prime of $S$ minimal over $K$, then there is a bond between $S / P$ and a prime factor of $R / I$. Since the minimal primes of $R / I$ are $Q / I$ and $Q^{\prime} / I$, it follows from (6.4) that $d(S / P) \leq \alpha$ for every minimal prime $P / K$ of $S / K$, and so $d(S / K) \leq \alpha$. It follows from (6.1) and (6.4) that if $T /(K \cap R)$ is a minimal prime of $R /(K \cap R)$, then $d(R / T) \leq \alpha$. We therefore conclude, again using (6.4), that $Q$ and $Q^{\prime}$ are primes minimal over $R \cap K$, and that $d(R /(R \cap K))=\alpha$. Moreover, there is a link from $Q /(R \cap K)$ to $Q^{\prime} /(R \cap K)$. We may therefore assume without loss of generality that $K=0$.

We are now in the situation where $d(R)=\alpha$ and $d(S) \leq \alpha$. If $P$ is a prime of $S$ lying over $Q$, then by (6.1) there is a bond from $S / P$ to $R / Q$. Hence, $d(S / P)=\alpha$ and $P$ is a minimal prime of $S$. Thus each prime of $S$ lying over $Q$ is a minimal prime of codimension $\alpha$, and similarly for each prime of $S$ lying over $Q^{\prime}$. In particular, since there exist primes of $S$ lying over $Q$ by (6.1), it holds that $d(S)=\alpha$. The theorem now follows from (6.3) and (6.5).

Remark. It is not necessarily the case for a finite extension of Noetherian rings $R \hookrightarrow S$ with $\operatorname{Tr}\left(S_{R}\right)=R$ that prime ideals of $S$ intersect to semiprime ideals of $R$. For example, let $S=M_{2}(k)$, where $k$ is a field, and let $R$ be the subring of matrices of the form $\left(\begin{array}{ll}a & 0 \\ b & a\end{array}\right)$. In this case there is a link from a prime ideal of $R$ to itself, and this link lifts to a trivial ideal link in $S$.

Example 6.7. Let $U$ be the enveloping algebra of a finite-dimensional semisimple Lie algebra over an algebraically closed field $k$ of characteristic zero. Then $U$ is a Noetherian domain of finite GK-dimension for which each module that is finite dimensional over $k$ is completely reducible. Let $G$ be a finite group of automorphisms of $U$, and let $A=U^{G}$. It can be deduced from [16] that (i) $U$ is finitely generated as a right and left $A$-module, (ii) $\operatorname{Tr}\left(U_{A}\right)=A$, (iii) each prime ideal of $U$ intersects to a semiprime ideal of $A$, and (iv) each right $A$-module finite dimensional over $k$ is completely reducible. 
Our approach above allows a somewhat more abstract result. Let $R$ be a Noetherian $k$-subalgebra of $U$ such that $U$ is finitely generated as a right $R$ module, such that prime ideals of $U$ intersect to semiprime ideals of $R$, and such that $\operatorname{Tr}\left(U_{R}\right)=R$. It follows from (6.6) that each right $R$-module finitedimensional over $k$ is completely reducible: Letting $d$ denote GK-dimension we see that $\{R, U\}$ possesses a symmetric dimension function; see [10] or [15]. It then follows from (6.6) and (6.1) that each link between co-finitedimensional maximal ideals of $R$ implies the existence of a link between cofinite-dimensional maximal ideals of $U$. However, a link between co-finitedimensional maximal ideals in a Noetherian ring occurs precisely when there is a nonsplit extension of the corresponding simple modules.

\section{REFERENCES}

1. A. D. Bell, Localization and ideal theory in Noetherian crossed products and differential operator rings, Ph.D. Dissertation, Univ. of Washington, 1984.

2. _ Notes on localization in noncommutative Noetherian rings, Cuadernos de Algebra 9, Universidad de Granada, 1988.

3. K. A. Brown and S. P. Smith, Bimodules over a solvable algebraic Lie algebra, Quart. J. Math. Oxford (2) 36 (1985), 129-139.

4. K. A. Brown and R. B. Warfield, Jr., The influence of ideal structure on representation theory, J. Algebra 116 (1988), 294-315.

5. B. Cortzen and L. W. Small, Finite extensions of rings, Proc. Amer. Math. Soc. 103 (1988), 1058-1062.

6. K. R. Goodearl and R. B. Warfield, Jr., An introduction to noncommutative Noetherian rings, London Math. Soc. Student Texts, no. 16, Cambridge Univ. Press, Cambridge, 1989.

7. T. J. Hodges and J. Osterburg, A rank two indecomposable projective module over a Noetherian domain of Krull dimension one, J. London Math. Soc. 19 (1987), 139-144.

8. A. V. Jategaonkar, Noetherian bimodules, primary decomposition, and Jacobson's conjecture, J. Algebra 71 (1981), 379-400.

9. __ Localization in Noetherian rings, London Math. Soc. Lecture Notes, no. 98, Cambridge Univ. Press, Cambridge, 1986.

10. G. R. Krause and T. H. Lenagan, Growth of algebras and Gelfand-Kirillov dimension, Pitman, London, 1985.

11. T. H. Lenagan, Artinian ideals in Noetherian rings, Proc. Amer. Math. Soc. 51 (1975), 499-500.

12. T. H. Lenagan and R. B. Warfield, Jr., Affiliated series and extensions of modules, J. Algebra (to appear).

13. E. S. Letzter, Primitive ideals in finite extensions of Noetherian rings, J. London Math. Soc. (2) 39 (1989), 427-435.

14. __ Prime ideals in finite extensions of Noetherian rings, J. Algebra 135 (1990), 412-439.

15. J. C. McConnell and J. C. Robson, Noncommutative Noetherian rings, Wiley-Interscience, New York, 1988.

16. S. Montgomery, Fixed rings of finite automorphism groups of associative rings, Lecture Notes in Math., vol. 818, Springer-Verlag, New York, 1980.

17. L. W. Small and R. B. Warfield, Jr., Finite extensions of rings. II, preprint, Univ. of California, San Diego.

18. L. Soueif, Normalizing extensions and injective modules, essentially bounded normalizing extensions, Comm. Algebra 15 (1987), 1607-1619.

19. R. B. Warfield, Jr., Bond invariance of G-rings and localization, Proc. Amer. Math. Soc. 111 (1991), 13-18.

Department of Mathematics, University of Washington, Seattle, Washington 98195 